\title{
Über die Fettsäuren aus Chloroplasten
}

\author{
Von Hildegard Debuch \\ Aus dem Physiologisch-chemischen Institut der Universität zu Köln \\ (Direktor: Prof. Dr. Dr. E. KLENK) \\ (Z. Naturforschg. 16 b, 246-248 [1961] ; eingegangen am 4. Januar 1961)
}

\begin{abstract}
In der vorliegenden Arbeit wurden die Gesamtfettsäuren der Lipoidextrakte reiner Chloroplasten von Antirrhinum majus Sippe 50, der Plastom-Mutante prasinizans * von Antirrhinum majus und von Allium porrum untersucht. Die quantitative Analyse der destillierten Fettsäuremethylester erfolgte mit Hilfe gaschromatographischer Methoden. Dabei zeigte sich, daß sich die Zusammensetzung der Fettsäuregemische von Chloroplasten verschiedener Arten praktisch nur in der mengenmäßigen Verteilung gleichartiger Fettsäuren voneinander unterscheidet.
\end{abstract}

Bereits 1942 berichteten Menke und Jacoв ${ }^{1}$ über orientierende Untersuchungen der Lipoide von Spinatchloroplasten-Substanz. Die zu der Zeit zur Verfügung stehenden Methoden gestatteten bei den vorhandenen Substanzmengen jedoch keine nähere Lipoidanalyse, obwohl man bereits auf reine Chloroplastenpräparate als Untersuchungsgut verzichtet hatte. Da inzwischen neue lipoidchemische Analysen mit relativ wenig Material durchgeführt werden können und die Gewinnung reiner Chloroplasten in ausreichenden Mengen möglich war, erschien es wünschenswert, zunächst ihre Fettsäuren auf gaschromatographischem Wege zu untersuchen.

Als Ausgangssubstanz dienten Lipoidextrakte** reiner Chloroplasten-Präparate von Antirrhinum majus Sippe 50 sowie der Plastom-Mutante prasinizans von Antirrhinum majus und von Allium porrum. Bei allen untersuchten Chloroplasten bestand das Gemisch zu $10-20 \%$ aus $\mathrm{C}_{16}$ zu $80-90 \%$ aus $\mathrm{C}_{18}$-Fettsäuren. Dabei ist auffallend, daß die Palmi- tinsäure praktisch als die einzige gesättigte Fettsäure auftritt, Stearinsäure findet sich nur zu $1,0-1,6 \%$ der Gesamtfettsäuren. Besonders bemerkenswert ist jedoch der außerordentlich niedrige Anteil der Ölsäure $(1,6-3,5 \%)$ an den Gesamtfettsäuren, da diese ungesättigte $C_{18}$-Fettsäure besonders weit verbreitet ist und reichlich in pflanzlichen Speicherorganen vorkommt. Dagegen stellt hier Linolensäure die Hauptkomponente dar $(44-71 \%)$.

Es lassen sich in der Zusammensetzung des Fettsäuregemisches deutliche Unterschiede zwischen den Chloroplasten verschiedener Arten aufweisen, die sich jedoch auf quantitative Differenzen einzelner Komponenten beschränken.

\section{Darstellung der Fettsäuren}

Die Isolierung der Chloroplasten erfolgte auf die bereits früher ${ }^{2}$ beschriebene Weise. Das gefriergetrocknete Material wurde bei Zimmertemperatur kurz in Methanol, anschließend in Äther suspendiert und abfil-

\begin{tabular}{|c|c|c|c|c|c|c|c|}
\hline \multirow{2}{*}{$\begin{array}{c}\text { Chloroplasten- } \\
\text { präparat }\end{array}$} & \multirow{2}{*}{$\begin{array}{c}\text { Gesamt- } \\
\text { lipoid- } \\
\text { extrakt } \\
{[\mathrm{g}]}\end{array}$} & \multicolumn{2}{|c|}{$\begin{array}{c}\text { Fettsäuremethylester }+ \\
\text { Unverseif bares }\end{array}$} & \multicolumn{2}{|c|}{ Unverseif bares } & \multicolumn{2}{|c|}{ freie Fettsäuren } \\
\hline & & {$[\mathrm{g}]$} & $\begin{array}{l}\text { in } \% \text { der } \\
\text { Ausgangs- } \\
\text { substanz }\end{array}$ & {$[\mathrm{g}]$} & $\begin{array}{l}\text { in \% der } \\
\text { Ausgangs- } \\
\text { substanz }\end{array}$ & {$[\mathrm{g}]$} & $\begin{array}{l}\text { in } \% \text { der } \\
\text { Ausgangs- } \\
\text { substanz }\end{array}$ \\
\hline $\begin{array}{l}\text { Antirrhinum } \\
\text { Antirrh. Mut. } \\
\text { Allium porrum }\end{array}$ & $\begin{array}{l}2,43 \\
0,69 \\
2,49\end{array}$ & $\begin{array}{l}1,38 \\
0,43 \\
1,56\end{array}$ & $\begin{array}{l}56,8 \\
62,4 \\
62,9\end{array}$ & $\begin{array}{l}0,43 \\
0,09 \\
0,30\end{array}$ & $\begin{array}{l}17,7 \\
13,0 \\
12,0\end{array}$ & $\begin{array}{l}0,76 \\
0,29 \\
0,96\end{array}$ & $\begin{array}{l}31,2 \\
42,0 \\
38,6\end{array}$ \\
\hline
\end{tabular}

Tab. l. Darstellung der Fettsäuren aus den Lipoidextrakten verschiedener Chloroplasten.

* Diese Mutante wurde von Herrn R. Maly im Max-Planck. Institut für Biologie, Abt. Melchers in Tübingen, gefunden. Die Chlorophyllsynthese erfolgt bei dieser Sippe nur zögernd.

1 W. Menke u. E. Jасов, Hoppe-Seyler's Z. physiol. Chem. 272, 227 [1942].
* Für die Überlassung der Präparate danke ich Herrn Prof. Dr. W. Menke (Botanisches Inst. d. Universität Köln) aufs herzlichste, sowie auch für die Unterstützung der Arbeit.

2 W. Kreutz u. W. Menke, Z. Naturforschg. 15 b, 402 [1960]. 
triert. Die vereinigten Extrakte engte man im Vakuum unter $\mathrm{N}_{2}$ bis zur Trockne ein und nahm den Rückstand in trocknem Äther auf. Dieser Lipoidextrakt wurde nach Abdampfen des Äthers mit dem 15-fachen Volumen 4-proz. methanolischer HCl 1 Stde. unter Rückfluß gespalten. Nach dem Abkühlen wurden die Fettsäuremethylester in Petroläther durch wiederholtes Ausschütteln aufgenommen, die petrolätherische Lösung wusch man mit Wasser neutral, trocknete sie über $\mathrm{Na}_{2} \mathrm{SO}_{4}$ und destillierte im Vakuum unter $\mathrm{N}_{2}$-Strom das Lösungsmittel ab. Anschließend verseifte man die Fettsäuremethylester durch 1-stdg. Kochen unter Rückfluß mit dem 30-fachen Volumen n/2-methanolischer $\mathrm{NaOH}$. Nach Stehen über Nacht im Eisschrank wurden die bei $+4^{\circ}$ festen Seifen abgesaugt, 3-mal in Petroläther (40 bis $60^{\circ}$ siedend) suspendiert und wieder abgesaugt. Von den flüssigen Seifen trennte man ebenfalls das Unverseifbare durch Ausschütteln in Petroläther ab.

Die petrolätherischen Lösungen wurden vereinigt und so lange mit Methanol-Wasser (Methanol : Wasser $=80: 20 ; 60: 40 ; 50: 50 \mathrm{v} / \mathrm{v}$ usw.) schließlich mit Wasser gewaschen, bis sich keine Emulsionen mehr bildeten. Nach dem Trocknen über $\mathrm{Na}_{2} \mathrm{SO}_{4}$ wurde aus der petrolätherischen Lösung nach Abdestillieren des Lösungsmittels das Unverseifbare gewonnen. Die festen Seifen und die methanolische $\mathrm{NaOH}$ wurden mit dem Methanol-Wasser vom Waschen der petrolätherischen Lösung vereinigt, mit verdünnter $\mathrm{HCl}$ angesäuert und 5-mal mit einer Lösung von Äther : Petroläther $1: 1$ ausgeschüttelt. Die vereinigten Ätherlösungen wusch man mit $\mathrm{H}_{2} \mathrm{O}$ neutral, trocknete sie über $\mathrm{Na}_{2} \mathrm{SO}_{4}$ und nach Abdestillieren des Lösungsmittels erhielt man die freien Fettsäuren.

\section{Analyse der Fettsäuren}

Nach dem Abtrennen des Unverseifbaren wurden die freien Fettsäuren wie oben beschrieben erneut in die Methylester übergeführt und diese zur Reinigung im Hochvakuum $\left(10^{-4}\right.$ Torr $)$ möglichst schnell nach Art einer Molekulardestillation in dem früher ${ }^{3}$ beschriebenen Apparat destilliert. Die destillierten Fettsäuremethylester wurden dann gaschromatographisch untersucht.

Chromatograph: Wheelco Instr. Div. Barber Colman Co Rockford Illinois Modell 10 Ionisations-Detektor.

\begin{tabular}{|l|c|c|c|}
\hline Chloroplastenpräparat & $\mathrm{C}_{14}$ & $\mathrm{C}_{16}$ & $\mathrm{C}_{18}$ \\
\hline Antirrhinum majus & - & 11,0 & 89,0 \\
Antirrhinum & & & \\
majus, Mutante & 0,7 & 16,2 & 83,1 \\
Allium porrum & 0,4 & 21,8 & 77,8 \\
\hline
\end{tabular}

Tab. 2. Zusammensetzung der hydrierten Fettsäuregemische (in \% der Gesamtfettsäuren).

3 H. Debuch, Hoppe-Seyler's Z. physiol. Chem. 304, 109 [1956].
Säule $120 \mathrm{~cm}$, Durchmesser $4 \mathrm{~mm}$, Stationäre Phase Reoplex 400. Trägermaterial: Celite (Perkin Elmer Präparat 098-1504, gewaschen und gesiebt). Mobile Phase: Argon. T-Säule: $187^{\circ}$; Zelle: $225^{\circ}$; Verdampfer: $273^{\circ}$. Die quantitative Auswertung der Gaschro-
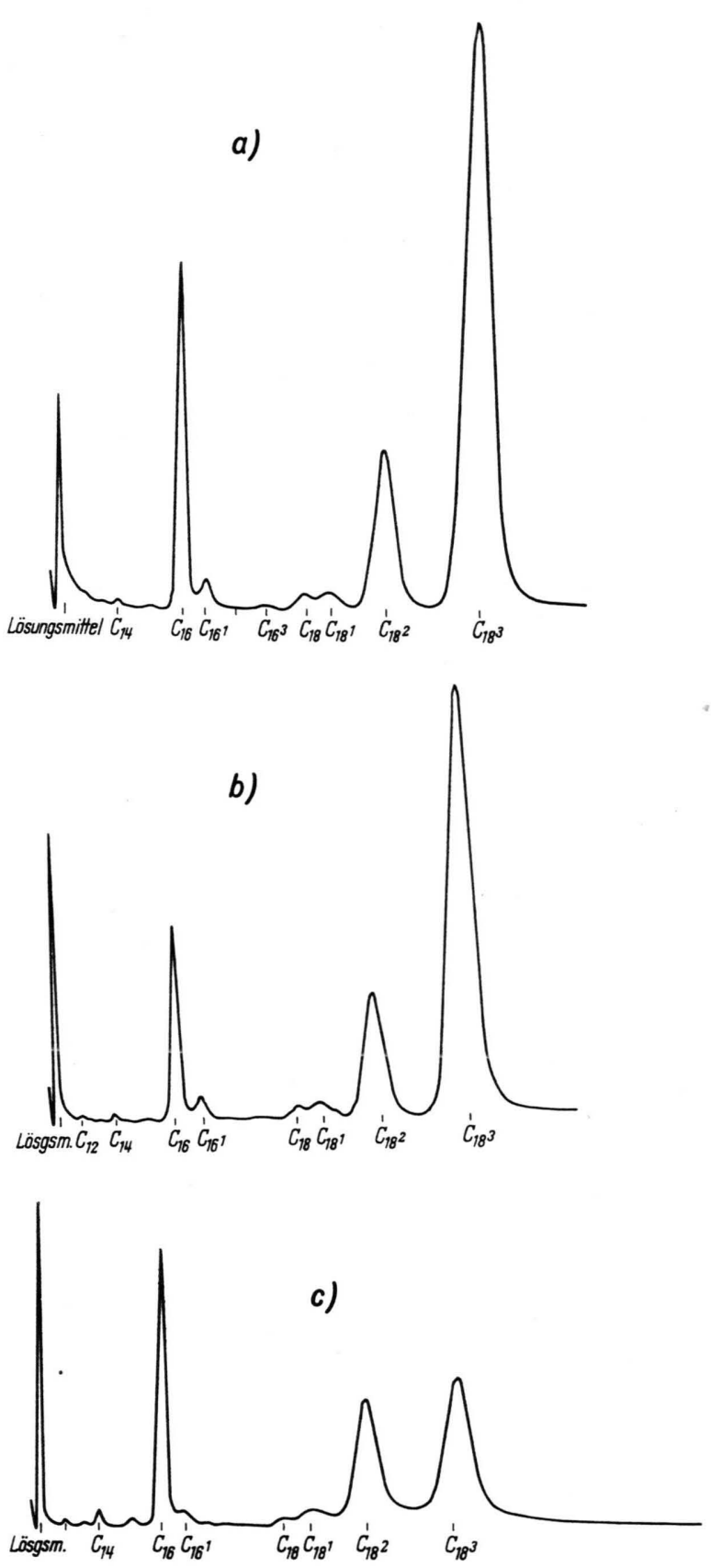

Abb. 1. Gaschromatographische Analyse der Fettsäuremethylester (in Aceton gelöst) nach der Destillation aus: a) Antirrhinum majus, b) Antirrhinum majus, Mutante, c) Allium porrum. 


\begin{tabular}{|c|c|c|c|c|c|c|c|c|}
\hline \multirow[b]{2}{*}{$\begin{array}{c}\text { Chloroplasten- } \\
\text { präparat }\end{array}$} & \multirow{2}{*}{$\begin{array}{c}\mathrm{C}_{14} \\
\text { Myri- } \\
\text { stin- } \\
\text { säure }\end{array}$} & \multicolumn{3}{|c|}{$\mathrm{C}_{16}$} & \multicolumn{4}{|c|}{$\mathrm{C}_{18}$} \\
\hline & & $\begin{array}{l}\text { Palmi- } \\
\text { tin- } \\
\text { säure }\end{array}$ & $\begin{array}{l}\text { Mono- } \\
\text { en- } \\
\text { säure }\end{array}$ & $\begin{array}{l}\text { Trien- } \\
\text { säure }\end{array}$ & $\begin{array}{l}\text { Stea- } \\
\text { rin- } \\
\text { säure }\end{array}$ & $\begin{array}{l}\text { Öl- } \\
\text { säure }\end{array}$ & $\begin{array}{l}\text { Linol- } \\
\text { säure }\end{array}$ & $\begin{array}{l}\text { Lino- } \\
\text { len- } \\
\text { säure }\end{array}$ \\
\hline $\begin{array}{l}\text { Antirrhinum majus } \\
\text { Antirrhinum majus, Mut. } \\
\text { Allium porrum }\end{array}$ & $\begin{array}{l}\text { Spur } \\
0,7 \\
0,4\end{array}$ & $\begin{array}{r}9,5 \\
13,8 \\
19,7\end{array}$ & $\begin{array}{l}1,3 \\
2,4 \\
2,1\end{array}$ & $\begin{array}{l}0,2 \\
- \\
-\end{array}$ & $\begin{array}{l}1,0 \\
1,6 \\
1,0\end{array}$ & $\begin{array}{l}1,6 \\
1,9 \\
3,5\end{array}$ & $\begin{array}{l}15,1 \\
15,0 \\
29,2\end{array}$ & $\begin{array}{l}71,3 \\
64,6 \\
44,1\end{array}$ \\
\hline
\end{tabular}

Tab. 3. Zusammensetzung der destillierten Fettsäuregemische (in \% der Gesamtfettsäuren) .

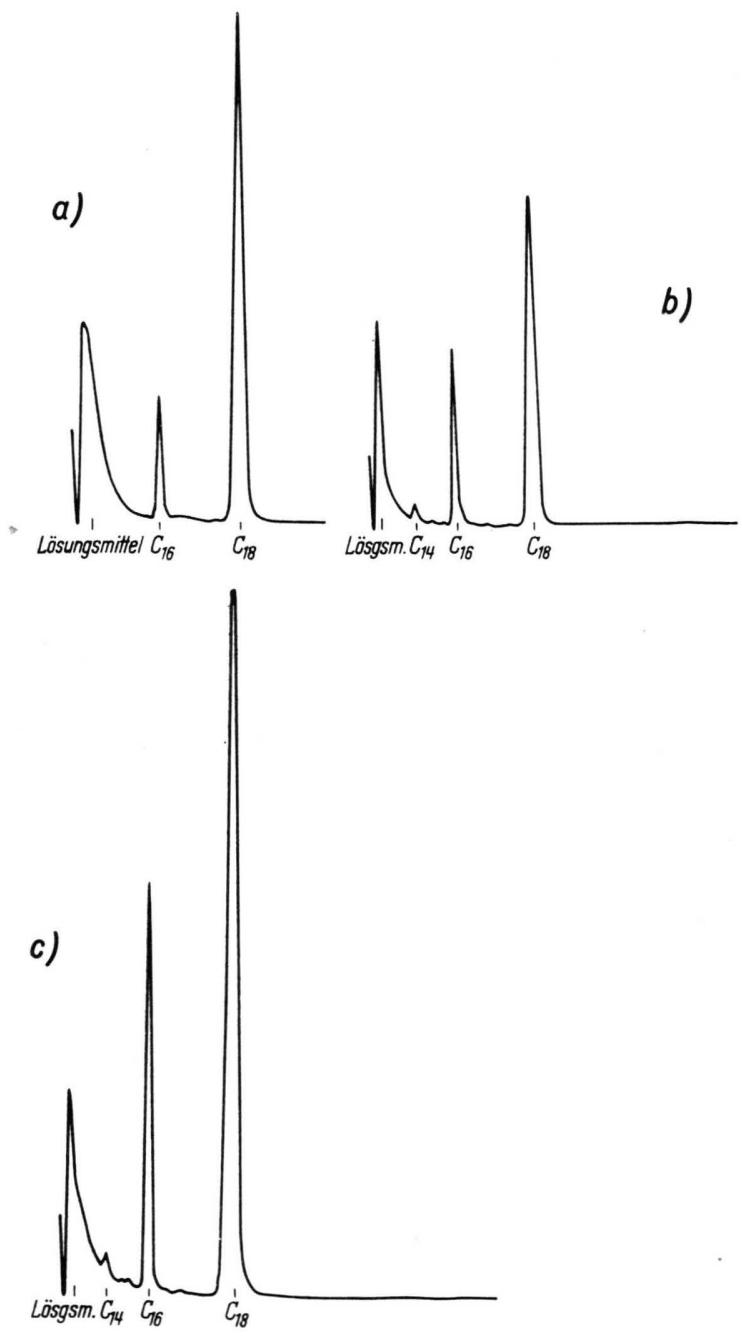

Abb. 2. Gaschromatographische Analyse der hydrierten Fettsäuremethylester (in Aceton gelöst) aus: a) Antirrhinum majus, b) Antirrhinum majus, Mutante, c) Allium porrum. T-Säule: $193^{\circ}$; Zelle: $245^{\circ}$; Verdampfer : $297^{\circ}$.

Übrige Daten: s. Abb. 1. matogramme der Gesamtfettsäuren wurde auf die am hiesigen Institut übliche Weise vorgenommen:

Eine Probe der destillierten Fettsäuremethylester wurde mit R a n e y - Nickel als Katalysator in Alkohol hydriert und wiederum gaschromatographisch untersucht (Abb. 2).

Durch Multiplikation des Flächeninhaltes unter den Kurven mit den für die gesättigten Fettsäuren (ebenfalls in Aceton gelöst) ermittelten Faktoren (Myristinsäure: 0,69; Palmitinsäure: 0,77; Stearinsäure: 1,00; Arachinsäure: 0,99) erhielt man jeweils den Anteil einer Fettsäurekomponente des hydrierten Gemisches.

Damit ist die prozentuale Verteilung der vorhandenen Fettsäuren in der Ausgangssubstanz entsprechend der Kettenlängen bestimmt. - Zur Berechnung aller Komponenten wurde der ermittelte Flächeninhalt aller Säuren der gleichen Kettenlänge aus den Gaschromatogrammen der nicht hydrierten Säuren (s. Abb. 1 a-c) anteilmäßig entsprechend der Menge mit analoger Kettenlänge im Gemisch der hydrierten Fettsäuren (Tab. 2) umgerechnet.

Mit Ausnahme der Substanz in Abb. 2 a $-\mathrm{c}$ als $\mathrm{C}_{18}$ bezeichnet, waren alle anderen Fettsäuremethylester durch Vergleichschromatogramme der entsprechenden Testsubstanzen identifizierbar. Eine $\mathrm{C}_{16}$-Tetraensäure weist nämlich unter diesen Bedingungen das gleiche Retentionsvolumen wie Stearinsäure auf. Deshalb wurden die destillierten Fettsäuremethylester am gleichen Chromatographen mit Apiezon (Präparat: Perkin Elmer) als stationärer Phase untersucht. T-Säule: $190^{\circ}$; Zelle: $237^{\circ}$; Verdampfer: $307^{\circ}$. Dabei verhalten sich die Retentionsvolumina anders als bei Reoplex 400 als stationärer Phase. Wie aus der Abb. 1 zu ersehen, ist das Retentionsvolumen z. B. der Stearinsäure kleiner als das der Ölsäure. Bei der Apiezonsäulenfüllung erscheinen die ungesättigten Fettsäuren gleicher Kettenlänge jedoch zusammen vor den entsprechenden gesättigten. In den oben beschriebenen $\mathrm{Gaschromatogrammen}$ war eindeutig Stearinsäure nachweisbar.

Die Arbeit wurde mit Unterstützung der D e u t schen Forschungsgemeinschaf turchgeführt. Frl. D. Bielefeld sei für ihre Mitarbeit gedankt. 